\title{
Konkurrierende Standards
}

\section{Soit ein paar Jahren existiert mit dem FSC-Siegel ein infernationaler Rahmen für ein Zertifizierungssystem für nachhaltig bewirtschaftetes Holz. Kürzlich hat sich eine Arbeitsgruppe über die Umsetzung in Deutschland geeinigt. Doch private Waldbesitzer sind hiermit unzufrieden und versuchen parallel, ein umstriftenes weiferes Siegel zu etablieren.}

$\mathrm{U}$ Von Jan Nill mweltverbände, Gewerkschaften und Vertreter der Forst- und Holzwirtschaft in Deutschland haben sich nach langen Verhandlungen geeinigt: Einstimmig wurden Mitte April Richtlinien für eine umweltverträgliche und sozial gerechte Forstwirtschaft verabschiedet. Diese entsprechen den international anerkannten Zertifizierungsprinzipien des Forest Stewardship Council (FSC). In dieser unabhängigen Organisation sind Forstwirtschaft, Umweltverbände und soziale Gruppen gleichberechtigt vertreten. Bis Ende 1998 wurden weltweit mehr als 12 Millionen Hektar Wald zertifiziert. Nach den vereinbarten Richtlinien gehören z.B. Kahlschläge, Chemieeinsatz und der Aufbau von Monokulturen der Vergangenheit an, die natürliche Verjüngung heimischer Baumarten hat Vorrang. Weiter werden lokale Interessen berücksichtigt, ein hoher Ausbildungs- und Sicherheitsstandard geprüft und ArbeitnehmerInnen möglichst ganzjährig beschäftigt (1).

In Deutschland haben sich bisher sieben Stadtwälder und 48 Gemeinden zur Bewirtschaftung ihres Waldes nach den Kriterien des FSC verpflichtet und dürfen ihre Produkte mit dem FSCGütesiegel bewerben. Über 40 deutsche Unternehmen haben sich auf Initiative des WWF als „Gruppe 98“ inzwischen für das globale ÖkoSiegel ausgesprochen und verpflichtet, mit zertifiziertem Holz bzw. Holzprodukten zu handeln. Darunter sind u.a. die Baumarktketten OBI und Praktiker, die Großdruckerei Mohndruck sowie der Otto-Versand. Wenngleich noch Probleme bei der Kontrolle des Standards bestehen und schon vereinzelt Betrugsfälle aufgedeckt wurden (2), sind somit prinzipiell die Voraussetzungen für eine verstärkte Verbreitung geschaffen.

Doch Holzverarbeiter und KonsumentInnen müssen sich in Zukunft wohl weiter zwischen mehreren Siegeln entscheiden: Ebenfalls im April hat die „Paneuropäische Initiative zur Zertifizierung nachhaltiger Waldbewirtschaftung“" (Pan Europ- ean Forest Certification, PEFC) einen Entwurf fuir ein europäisches (Rahmen-) Siegel vorgestellt. Dahinter stehen private europäische Waldbesitzer aus mehreren Ländern die ihren Einfluß in den FSC-Gremien als zu gering erachten. Das Kernelement ist hier die unabhängige Überprüfung und Zertifizierung nachhaltiger Forstwirtschaft auf regionaler Ebene, da eine einzelbetriebliche Prïfung aufgrund des kleinstrukturierten Waldbesitzes in Europa für zu kostspielig gehalten wird. Das PEFC-System wird in Deutschland zur Zeit in drei Modellprojekten erprobt. Nach einem breiteren Diskussionsprozeß, der weitere Akteure einbezieht, soll im Verlauf des Sommers dann die Gründung des Zertifikats erfolgen (3).

Bei den Umweltverbänden stößt das PEFC-Siegel hingegen auf scharfe Kritik. BUND, Greenpeace, NABU, Robin Wood und WWF bemängeln neben dem bisher fehlenden Einbezug von Umweltorganisationen in den Prozeß drei weitere Punkte. Sie sehen erstens keine Anzeichen, daß das Zertifikat ökologische Verbesserungen für den Wald bringt. Zweitens halten sie die Zertifizierung von ganzen Regionen für wenig glaubwürdig. Schließlich fordern sie, daß ein Gütesiegel weltweit gïltig sein sollte. Sie sprechen sich daher, wie auch die europäischen Umweltverbände generell, für das FSC-Siegel aus und betonen, daß in diesem Rahmen prinzipiell auch das PEFC-Zertifikat anerkannt werden könnte, sofern es die ökologischen Mindeststandards des FSC erfiillt (4).

\section{Anmerkungen}

(1) Nähere Informationen hierzu gibt es bei der FSCArbeitsgruppe Deutschland, Stefan Lutz, Postfach 700104, 79055 Freiburg, Tel. 0761/ 69664-33, Fax -34. Den in der Arbeitsgruppe vertretenen Waldbesitzern gehören etwa zehn Prozent der deutschen Forste.

(2) Vgl. z.B. Die Tageszeitung vom 18./19.7.1998, S. 7. (3) Ansprechpartner in Deutschland ist der Bayerische Waldbesitzerverband, Hans Baur, Tel. 089/ 580-3089, Fax -7015.

(4) Die Federführung hat hier der WWF Deutschland, Frau von Zitzewitz, Tel. 069/ 79144-0.

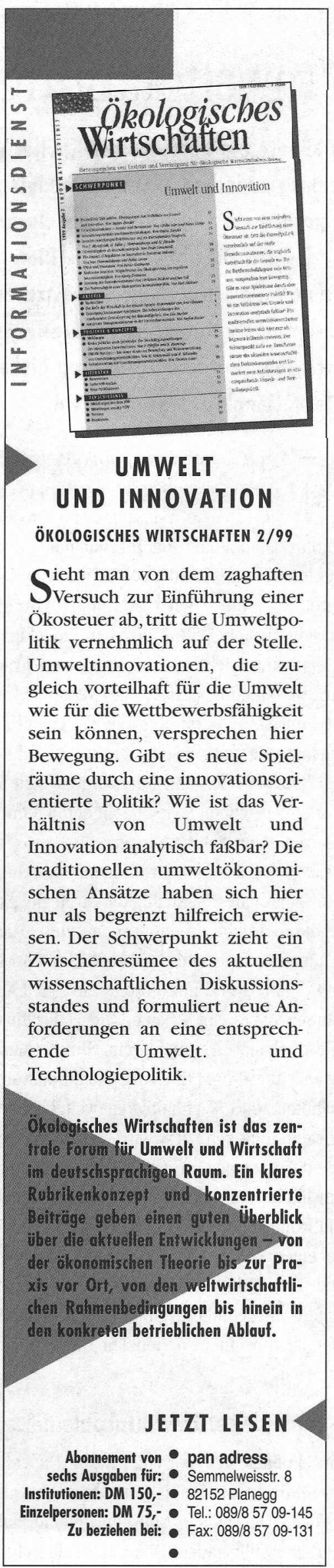


(c) 20I0 Authors; licensee IÖW and oekom verlag. This is an article distributed under the terms of the Creative Commons Attribution Non-Commercial No Derivates License (http://creativecommons.org/licenses/by-nc-nd/3.o/), which permits unrestricted use, distribution, and reproduction in any medium, provided the original work is properly cited. 